\title{
Correction to: Budget Impact Analysis of Brivaracetam Adjunctive Therapy for Partial-Onset Epileptic Seizures in Valencia Community, Spain
}

\author{
Isabel Barrachina-Martínez ${ }^{1}$ (D) David Vivas-Consuelo ${ }^{1}$ - Anna Piera-Balbastre ${ }^{2}$
}

Published online: 20 February 2018

(C) Springer International Publishing AG, part of Springer Nature 2018

\section{Correction to: Clin Drug Investig}

https://doi.org/10.1007/s40261-017-0615-z

In the original publication, the abstract, conclusion was incorrectly published.

Abstract, Conclusions which previously read:

Brivaracetam is a therapeutic alternative that allows savings for the health system in patients with non-controlled epilepsy when used as monotherapy, having a fixed, predictable annual cost (independent of dose) from the first day of treatment as the lack of need for titration means the patient is within a range of therapeutic doses from the first dose.

\section{Should read:}

Brivaracetam is a therapeutic alternative that allows savings for the health system in patients with non-controlled epilepsy in monotherapy, having a fixed, predictable annual cost (independent of dose) from the first day of treatment as the lack of need for titration means the patient is within a range of therapeutic doses from the first dose.

The original article was corrected.

The original article can be found online at https://doi.org/10.1007/ s40261-017-0615-z.

Isabel Barrachina-Martínez

ibarrach@upvnet.upv.es

Departament of Economics and Social Sciences, Research

Centre for Health Economics and Management, INECO,

Universitat Politècnica de València, Edificio $7 \mathrm{~J}$, Camino de Vera s/n, 46022 Valencia, Spain

2 Department of Neurology, Hospital Clínico Universitario de Valencia, Valencia, Spain 